\title{
Observation of a Neutrino Burst in Coincidence with Supernova 1987A in the Large Magellanic Cloud
}

\author{
R. M. Bionta, ${ }^{(12)}$ G. Blewitt, ${ }^{(4)}$ C. B. Bratton, ${ }^{(5)}$ D. Casper, ${ }^{(2,14)}$ A. Ciocio, ${ }^{(14)}$ R. Claus, ${ }^{(14)}$ B. Cortez, ${ }^{(16)}$ \\ M. Crouch, ${ }^{(9)}$ S. T. Dye, ${ }^{(6)}$ S. Errede, ${ }^{(10)}$ G. W. Foster, ${ }^{(15)}$ W. Gajewski, ${ }^{(1)}$ K. S. Ganezer, ${ }^{(1)}$ \\ M. Goldhaber, ${ }^{(3)}$ T. J. Haines, ${ }^{(1)}$ T. W. Jones, ${ }^{(7)}$ D. Kielczewska, ${ }^{(1,8)}$ W. R. Kropp, ${ }^{(1)}$ J. G. Learned, ${ }^{(6)}$ \\ J. M. LoSecco, ${ }^{(13)}$ J. Matthews, ${ }^{(2)}$ R. Miller, ${ }^{(1)}$ M. S. Mudan, ${ }^{(7)}$ H. S. Park, ${ }^{(11)}$ L. R. Price, ${ }^{(1)}$ \\ F. Reines, ${ }^{(1)}$ J. Schultz, ${ }^{(1)}$ S. Seidel, ${ }^{(2,14)}$ E. Shumard, ${ }^{(16)}$ D. Sinclair, ${ }^{(2)}$ H. W. Sobel, ${ }^{(1)}$ J. L. Stone, ${ }^{(14)}$ \\ L. R. Sulak, ${ }^{(14)}$ R. Svoboda, ${ }^{(1)}$ G. Thornton, ${ }^{(2)}$ J. C. van der Velde, ${ }^{(2)}$ and C. Wuest ${ }^{(12)}$ \\ ${ }^{(1)}$ The University of California, Irvine, Irvine, California 92717 \\ ${ }^{(2)}$ The University of Michigan, Ann Arbor, Michigan 48109 \\ ${ }^{(3)}$ Brookhaven National Laboratory, Upton, New York 11973 \\ ${ }^{(4)}$ California Institute of Technology, Jet Propulsion Laboratory, Pasadena, California 91109 \\ ${ }^{(5)}$ Cleveland State University, Cleveland, Ohio 44115 \\ ${ }^{(6)}$ The University of Hawaii, Honolulu, Hawaii 96822 \\ ${ }^{(7)}$ University College, London WCIE 6BT, United Kingdom \\ ${ }^{\left({ }^{8}\right)}$ Warsaw University, Warsaw, Poland \\ ${ }^{(9)}$ Case Western Reserve University, Cleveland, Ohio 44106 \\ ${ }^{(10)}$ The University of Illinois, Urbana, Illinois 61801 \\ ${ }^{(11)}$ The University of California, Berkeley, California 94720 \\ ${ }^{(12)}$ Lawrence Livermore National Laboratory, Livermore, California 94550 \\ ${ }^{(13)}$ The University of Notre Dame, Notre Dame, Indiana 46556 \\ ${ }^{(14)}$ Boston University, Boston, Massachusetts 02215 \\ ${ }^{(15)}$ Fermi National Accelerator Laboratory, Batavia, Illinois 60510 \\ ${ }^{(16)}$ AT\& T Bell Laboratories, Summit, New Jersey 07910
}

(Received 13 March 1987)

\begin{abstract}
A burst of eight neutrino events preceding the optical detection of the supernova in the Large Magellanic Cloud has been observed in a large underground water Cherenkov detector. The events span an interval of $6 \mathrm{~s}$ and have visible energies in the range 20-40 MeV.
\end{abstract}

PACS numbers: $97.60 . \mathrm{Bw}, 14.60 . \mathrm{Gh}, 95.85 . \mathrm{Sz}$

According to conventional supernova theory, ${ }^{1}$ the recently observed supernova explosion ${ }^{2}$ (SN1987A) in the nearby Large Magellanic Cloud should have released approximately $3 \times 10^{53}$ ergs of gravitational binding energy in a burst consisting of $\sim 10^{58}$ neutrinos in a time interval of a few seconds. In this Letter, we report the observation of a burst of neutrino events, completely contained within the volume of the IMB (Irvine-MichiganBrookhaven) water Cherenkov detector, occurring close to the estimated time of the supernova collapse. The observed signal consists of eight neutrinos with energies in the range of 20-40 MeV, spaced over an interval of $6 \mathrm{~s}$. The background rate of contained events from cosmicray-produced neutrinos interacting in this detector is $\sim 2 / \mathrm{d}$ in the range $20-2000 \mathrm{MeV}$.

The IMB detector, designed to search for proton decay, is located in the Morton-Thiokol salt mine near Fairport, Ohio $\left(41.7^{\circ} \mathrm{N}, 81.3^{\circ} \mathrm{W}\right)$ at a depth of $1570 \mathrm{~m}$ water equivalent. It consists of a rectangular tank $\left(22.5 \times 17 \times 18 \mathrm{~m}^{3}\right)$ filled with purified water. The active volume for this search is approximately 5000 metric tons compared to the nominal fiducial volume of 3300 metric tons used in the proton-decay search. The six sides are instrumented with 2048 8-in. photomultiplier tubes (PMT's) arranged on an approximate 1-m grid. The tubes are mounted on wave-shifter plates, which increases their light-collection efficiency by a factor of 2 . Each tube provides timing and pulse-height information, from which the vertex, direction, and energy of a charged particle track may be reconstructed. ${ }^{3}$ For a 7 -h period of time containing the observed neutrino burst, one-quarter of the PMT's, representing essentially one contiguous region of the detector's walls, were not operational because of the failure of one of four high-voltage power supplies. The absence of these PMT's has been taken into account for all the results reported here.

The detector is triggered when at least 25 PMT's fire in $50 \mathrm{~ns}$; this corresponds to about 40 or more PMT's firing in the 511-ns trigger-recording window. Typically about 15 PMT's fire in this window as a result of random noise. The trigger efficiency as a function of visible energy has been determined and is given in Table $I$. Cosmic-ray muons trigger the detector at a rate of $\sim 2.7$ $\mathrm{Hz}$. Neutrino events are distinguished from cosmic-ray muon events by the fact that the reconstructed vertex of a neutrino interaction will occur within the detector volume (contained event) while that of a cosmic-ray muon will occur at the top or walls of the detector and the direction will be downward. As previously stated, the number of neutrino interactions is $\sim 2 / \mathrm{d}$. 
TABLE I. The estimated trigger efficiency for the detector with the loss of one high-voltage power supply as a function of visible energy.

\begin{tabular}{cc}
\hline \hline $\begin{array}{c}\text { Visible energy } \\
(\mathrm{MeV})\end{array}$ & Trigger efficiency \\
\hline 20 & 0.14 \\
30 & 0.56 \\
40 & 0.76 \\
50 & 0.89 \\
60 & 0.92 \\
\hline \hline
\end{tabular}

The absolute time of an event in universal time (UT) is recorded to $\pm 50 \mathrm{~ms}$ by means of a WWVB clock; the relative time for each event is known to better than $1 \mathrm{~ms}$.

The signal of a supernova is expected to be a burst of low-energy neutrinos occurring over a period of seconds. In the search for low-energy neutrino events, an upper limit of 100 PMT's firing was set as an off-line acceptance criterion. To search for such a burst of neutrinos, a 6.4-h period starting at UT 23 February $5^{\text {h }} .00^{\mathrm{m}} 00^{\text {s }}$. (when one-quarter of the PMT's were off) was selected and divided into nonoverlapping 10-s intervals. The number of events with fewer than 100 PMT's was determined for each 10-s interval; the frequency distribution is given in Table II. For $N \leq 5$, this distribution is fitted well by a Poisson distribution with $\langle N\rangle=0.77$ events per 10 -s interval. However, one interval, (UT) $7^{\mathrm{h}}$. $35^{\mathrm{m}}$. $40^{\mathrm{s}}$. to 7 h $35^{\mathrm{m}} .50^{\mathrm{s}}$., is found to contain nine events. A search of $\sim 60 \mathrm{~h}$ around this interval revealed no other $10-\mathrm{s}$ interval with more than six events.

With use of the track-imaging capabilities of this detector, the nine events during this time interval were studied in detail to determine if they could be due to charged particles entering the detector, or possibly spurious triggers of instrumental origin. One event was identified as a typical cosmic-ray muon. The remaining
TABLE II. The frequency distribution of events in 10-s intervals of the 6.4-h period containing the neutrino burst.

\begin{tabular}{cc}
\hline \hline No. of events & No. of 10 -s intervals \\
\hline 0 & 1043 \\
1 & 860 \\
2 & 307 \\
3 & 78 \\
4 & 15 \\
5 & 3 \\
6 & 0 \\
7 & 0 \\
8 & 0 \\
9 & 1 \\
$\geq 10$ & 0 \\
\hline \hline
\end{tabular}

eight events are identified as neutrino interactions in the detector volume; they occur within an interval of less than $6 \mathrm{~s}$. None of the events can be identified as a muon since no decay electron followed. We found no other neutrino events during this 6.4 -h period.

The visible energies corresponding to these events were determined by our correcting the observed PMT pulse heights for noise and geometric effects and comparing with a detailed simulation of Cherenkov light production in the detector. We further note that the light levels are comparable to those observed from muon-decay electrons, which were used as an independent energy calibration. If these events are caused by electron antineutrinos on free protons, then the visible energy corresponds closely to the neutrino energy. The uncertainties in these neutrino energy determinations are estimated to be $\pm 25 \%$ (statistical plus systematic). Reconstruction of the individual track directions has an uncertainty of about $\pm 15^{\circ}$. The observed events, times of occurrence, number of PMT's firing, estimated energies, and track angles with respect to the supernova are given in Table

TABLE III. Characteristics of the contained neutrino events recorded on 23 February.

\begin{tabular}{ccccc}
\hline \hline Event No. & $\begin{array}{c}\text { Time } \\
\text { (UT) }\end{array}$ & No. of PMT's & $\begin{array}{c}\text { Energy } \\
(\mathrm{MeV})\end{array}$ & $\begin{array}{c}\text { Angular distribution } \\
\text { (degrees) }\end{array}$ \\
\hline 33162 & $7: 35: 41.37$ & 47 & 38 & 74 \\
33164 & $7: 35: 41.79$ & 61 & 37 & 52 \\
33167 & $7: 35: 42.02$ & 49 & 40 & 56 \\
33168 & $7: 35: 42.52$ & 60 & 35 & 63 \\
33170 & $7: 35: 42.94$ & 52 & 29 & 40 \\
33173 & $7: 35: 44.06$ & 61 & 37 & 52 \\
33179 & $7: 35: 46.38$ & 44 & 20 & 39 \\
33184 & $7: 35: 46.96$ & 45 & 24 & 102 \\
\hline \hline
\end{tabular}

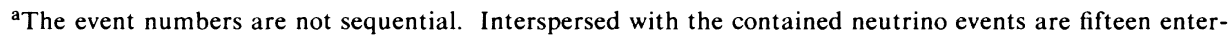
ing cosmic-ray muons.

${ }^{b}$ Error in energy determination is $\pm 25 \%$ (systematic plus statistical)

${ }^{c}$ Individual track reconstruction uncertainty is $15^{\circ}$. Note that this angular distribution will be systematically biased toward the source because of the location of the inoperative PMT's 
III. As a result of the missing PMT's, there is a bias against the detector triggering on tracks moving toward the Large Magellanic Cloud; the quantitative extent of this bias is under investigation. Individual track directions, however, are unaffected by this bias.

The detector is dead for about $35 \mathrm{~ms}$ after each trigger and the total dead time during the 6 -s burst was measured to be $0.8 \mathrm{~s}$. When corrected for dead-time and trigger losses, our observation corresponds to 22 events in the 6-s interval. If we assume, for simplicity, monoenergetic $32-\mathrm{MeV} \bar{v}_{e}$ 's interacting via inverse beta decay on free protons with a cross section of $8 \times 10^{-41} \mathrm{~cm}^{2}$, this corresponds to a total flux of $8 \times 10^{8} \mathrm{~cm}^{-2}$. The total neutrino output from the supernova is then $3 \times 10^{56}$ corresponding to a luminosity of $\bar{v}_{e}$ of $1 \times 10^{52}$ ergs above our threshold; the flux and luminosity have an estimated uncertainty of a factor of 2 .

In addition to the systematic search over the 60-h period, a more detailed search was performed for the included period UT 23 February $2^{\mathrm{h}}$. $22^{\mathrm{m}}$. to $3^{\mathrm{h}}$. $22^{\mathrm{m}}$, encompassing the time $2^{\mathrm{h}}$. $52 \mathrm{~m}$ at which a neutrino burst was reported ${ }^{4}$ to have occurred in the liquid scintillation detector of the Mount Blanc Neutrino Observatory. Our detector was fully operational during this period. A visual scan of all triggers with fewer than 100 PMT's yielded no contained events; this implies a 90\%-confidence-level upper limit on the $\bar{v}_{e}$ flux above $20 \mathrm{MeV}$ during this time of $2 \times 10^{8} \mathrm{~cm}^{-2}$. The subsequent selection of the $6.4-\mathrm{h}$ period in which the observed neutrino burst was found came after our learning of the detection of a neutrino signal by Hirata et al. ${ }^{5}$ at UT $7{ }^{\mathrm{h}} .35^{\mathrm{m}} 35^{\mathrm{s}}$. with an uncertainty of $\pm 1 \mathrm{~min}$.
Our events and their proximity in time to the optical observation of the supernova $1987 \mathrm{~A}$ are compelling evidence that neutrinos have been seen from a supernova collapse. It is clear that these data can be used to study fundamental properties of supernovae and neutrinos; further calculations are in progress.

This work was supported in part by the U.S. Department of Energy. We acknowledge the assistance of $R$. Becker-Szendy in the analysis of the data, and the vital assistance of J. Bastulli, T. Darden, E. Hazen, J. Hise, C. Mailander, J. Reese, and R. Render for maintaining and operating the detector. The hospitality of the Morton-Thiokol company, in whose Fairport Mine these events were recorded, is also appreciated.

${ }^{1}$ S. A. Colgate and R. H. White, Astrophys. J. 143, 626 (1966); J. R. Wilson et al., Ann. N.Y. Acad. Sci. 470, 267 (1986); J. N. Bahcall, A. Dar, and T. Piran, Nature 326, 155 (1987); T. Walker and D. Schramm, Fermilab Report No. 86/133A, 1986 (to be published); S. A. Bludman and P. J. Schinder, University of Pennsylvania Report No. UPR 0326T, 1987 (to be published).

${ }^{2}$ W. Kunkel and B. Madore, IAU Circular No. 4316 (24 February 1987).

${ }^{3}$ Further details concerning this detector can be found in R. M. Bionta et al., Phys. Rev. Lett. 51, 27 (1983); B. G. Cortez et al., Phys. Rev. Lett. 52, 1092 (1984); T. J. Haines et al., Phys. Rev. Lett. 57, 1986 (1986).

${ }^{4} \mathrm{M}$. Aglietta et al., "Characteristics of a Neutrino Burst Detected During the Occurrence of Supernova 1987A" (to be published); IAU Circulars No. 4323 and No. 4332.

${ }^{5} \mathrm{~K}$. Hirata et al., preceding Letter [Phys. Rev. Lett. 58, 1490 (1987)]. 